\section{RELATIVIST PSYCHOLOGY: A NEW CONCEPT OF PSYCHOLOGICAL MEASUREMENT}

Anatoly P. Suprun

Institute of systems analysis, Russian Academy of Sciences

Moscow

The article demonstrates the inadequacy in the traditional approach of psychology to the description of products of mental representation of perception. The principles of objective representation of subjective experience (principles of "mental map" construction) contain a whole range of contradictions. We propose a consistent mathematical description of the "mental map" based on psychosemiotic principles. Instead of the classical space of characteristics/properties we introduce semantic space that opens the way to a truly adequate representation of psychic reality. The article discusses metrics of the mental map, as well as substantiates the possibility of applying conservation laws to psychology. It is shown that it is possible to reach an adequate representation of research object in the humanities, equivalent to that of natural sciences in terms of accuracy. To verify a new approach, we make use of our theoretical deductions in the explanation of experimental data widely known in psychophysics and inconsistent with Stevens law.

Keywords: Relativist psychology, mental representation, mental map, psychosemiotic, semantic space, psychophysic.

\section{Representation and Description}

\section{of Experience in Classical Theory}

We believe that the problems psychology faces in its development as a valid scientific theory are, first of all, not related to the complexity of psyche itself as a research subject (to which psychologists make frequent references). They are more probably related to the inadequate representation of psychological phenomena in terms of theoretical concepts. Any experience is initially psychological, as it is related to the psychical process of perceiving reality (physical, social etc.), and therefore, basic rules of its theoretical representation must be universal to all sciences. Below we will

Preparation of the paper was supported by grant of The paper Russian Foundation of Fundamental Research (RFFI Project 08-06-001-176a). show that adequate semiotic representation of any type of experience is universal, and it allows introduction of semantic laws of conservation similar to those in physics. It makes possible turning psychology into a discipline equivalent to exact sciences in terms of prediction potential. In this connection, it results in changes in the approach to the construction of psychological scales and conduction of psychological measurements. Finally, we test theoretical proposals on the basis of Stevens law analysis.

We issue from the fact that the subject imagines external reality in a specific form through sensations. I. P. Pavlov called this called this form the first signal system (Pavlov, 1952, p. 215) - the psychic reflection of outside reality. In fact, this is an individual mental map of the "outside" world located "to the other side" of our sensations (Suprun, 2001). An individual correlates all his actions with that map, the actions aimed at satisfaction of his vital needs. Evidently enough, the more adequate and full the reflection of outside reality in the mental map, the more successful will be the process of an individual's adaptation to the environment and orientation therein. It should be specified that in our understanding the mental map presupposes only the result of the psychic process of reality reflection, as different from the processes themselves (perception, sensation, thought, memory, etc.)

The subject receives information on the interaction of his organism with the outside reality in the form of sensations (signals). Thereby he correlates modalities of different sensations with quality, whereas their intensity - with quantity. For example, sound (quality) can be soft and loud in terms of intensity; light (quality) can be bright or dim in terms of intensity; etc. It is evident that intensity may be represented in a quantitative form (for example, measured in decibels, luxes, etc.).

So, the subject has nothing but sensations and no way to find out what is there to the other side of sensations. The most important thing is to be able to efficiently orient oneself in the world in search of resources required to satisfy vital needs.

Thus, Reality is given to us as subjective sensations that code certain "properties" of the outside world. The objective character of Reality would be reflected in the efficiency, with which we are able to satisfy our needs, according to the model (mental map) of the world we have managed to construct on the basis of our sensations in the process of adaptation to the world. Complication of existence conditions and development of requirements bring about evolving of new secondary properties, semantically more complex, and essential for world description and orientation. 
Genetically determined mechanisms of adaptation to environment allow the individual to trace stable relationships between various sensations, correlate them with object properties and conditions for satisfying his requirements. The result of this process can be reconstructed in the form of a mental map, in compliance with the world image we managed to create on the basis of our sensations, when adapting to the world. Resemblance of classes of sensations, vital requirements, existence conditions and mechanisms of adaptation to environment creates basic prerequisites for generic similarity of mental maps and, therefore, possibilities for intraspecific communication, which received maximal development by humans, having brought about the second signal system (Pavlov, 1952, p. 215) or language. Further unification of mental maps does not only occur due to generic and territorial factors, but also linguistic, and further, social, cultural and economic factors. Yet all the same factors lead to definite differences within specific communities. The unification peculiarity in socially grounded components of mental maps of various communities determines mentality.

Thus, the second signal system according to Pavlov is a translation of the first signal system (mental map) into semiotic form. In fact, by using the verbal representation of reality, science does not study the world itself, but its mental reflection - our image of it. And different branches of science employ their specific conceptual systems for it. Yet, in one way or another, all of them perform linguistic modeling of our image of Reality. It is natural, since language is a universal tool for description of any phenomenon in all sciences, language being a semiotic system that is to give an adequate representation of any type of object with the help of the mode of reality perception natural to a given mentality. Therefore, it is the semiotic axioms and rules determine what can be presented in a theory ${ }^{1}$. Below we will discuss the issue of the kind of restrictions to our reality representation, caused by the way of its description.

\footnotetext{
${ }^{1}$ The famous theoretical physicist Werner Heisenberg commented on this problem in the following way: "understanding of any kind, whether it be scientific or not, depends on our language, on the fact that we can transmit our ideas. Every instance of describing phenomena, experiments and their results is also based on the language as the only way of understanding, recognition. The words of that language express concepts of everyday life, which in the scientific language of physics can be more accurately defined to the point of classical physical notions... As soon as a physicist rejected that basis, he would lose the opportunity for unambiguous explanation, and he would not be able to further develop his science (Heisenberg, 1989, p. 87-88)
}

\section{Specification of Semantic Space}

and Relativist Correlations

Since the mental map is our semiotic representation of Reality (or its virtual model, according to which we are oriented in the environment), then, to perceive the laws of psyche and individual behavior, it is important, as a minimum, to reconstruct relevant fragments of this map. Issuing from the above argumentation, the principles of mental map construction must necessarily contain the following grounds:

1. A theoretical equivalent of the mental map (concept) must give an adequate ${ }^{2}$ semiotic representation in a certain space of characteristics. Such a space of characteristics (properties) presupposes the adequate, full-value, unambiguous description of phenomena.

2. Since we propose a formal semiotic method of representing any nature of objects in the mental map, it must be universal, i.e. applicable in both the humanities and natural sciences.

Classical theory performs description of any object ${ }^{3}$ (phenomenon) through enumerating its properties (qualities) and indicating their intensities $^{4}$ (the degree of those qualities' expressedness), that is why a formal equivalent definition of object $\Omega$ can be put down in vector form as: $\vec{U}=\left\{Q_{1}, Q_{2}, \ldots, Q_{n}\right\}^{5}$, where coordinate $Q_{j}$ - intensity of the $j$-th property

${ }^{2}$ If the original conceptualization of phenomena is inadequate, it inevitably affects subsequent conclusions, and one cannot expect more or less valid forecasts from such a theory. Further we will demonstrate that classical description of psychical phenomena does not satisfy the abovementioned requirements

${ }^{3}$ From the point of view of semantics, objects are no more than a convenient way of describing steadily repeating sensation complexes under certain conditions. From the formal point of view, an object is a factor, connecting characteristics in a certain respect. When the conditions that determine relation of these characteristics, disappear, the object itself "disappears" (changes).

${ }^{4}$ An estimation scale of each characteristic may be assigned by a segment of a straight line with marked ends (for example, "light-heavy", "kind-evil" etc.) and a marked neutral point. Zero or middle scale mark determines the most probable, "customary" intensity value of a characteristic in a given population, as a customary, monotonous irritant ceases to cause orientation reaction in a human organism, and it is practically imperceptible on the conscious level.

${ }^{5}$ Here each vector coordinate corresponds to the intensity of a specific property. For example, intensity of psychological qualities of a personality: $Q_{1}$ - points for extroversion, $Q_{2}$ - points for neuroticism, etc. 
$(j=1,2, \ldots, n)$. Thus, any object $\Omega$ could be definitely correlated to a vector $\vec{U}$ (here and further a vector is marked by an arrow above sign) in a property space, i.e. $\vec{U} \rightarrow \Omega$. Normally, Euclidean metrics of this space is presupposed ${ }^{6}$.

Let us try to demonstrate that such a representation (accepted in psychology and sociology) is not adequate to Reality ${ }^{7}$.

First, let us take a specific example. To make it simple, let us suppose that we are studying an object, described through only three characteristics: $\vec{U}=\left\{Q_{1}, Q_{2}, Q_{3}\right\}$, for example - a cup of coffee that we are having, while inside a moving automobile ${ }^{8}$. For definiteness, let $Q_{1}$ be the speed of our cup (or intensity of automobile movement), $Q_{2}$ - intensity of color (taste, smell, etc.), $Q_{3}$ - mass (in physics it is to be understood as the degree of inertness related to speed $-Q_{1}$ ). If we simply pour two cups of coffee together, then the composition of two identical objects $\left(\vec{U}=\vec{U}^{\prime}\right)$ would yield the following result according to the rules of vector composition:

$$
\vec{U}_{\Sigma}=\vec{U}+\vec{U}^{\prime}=\left\{2 Q_{1}, 2 Q_{2}, 2 Q_{3}\right\} .
$$

Yet the speed $Q_{1}$ must remain unchanged. Color (taste, smell) intensity will not change either. Mass will be the only quality that doubles after the composition of two identical bodies. Hence the conclusion: object mass is not a property in the sense we are applying the term to such qualities as speed, color, etc., since it changes according to different laws ${ }^{9}$. In fact, mass does not reflect the intensity of a certain quality, but the quantity of that quality or its resistance to change (inertness or rigidity). Indeed, a new object $\vec{U}_{\Sigma}$ has not changed either speed, or color, or smell. Yet to "exhaust" its properties, it would take twice as much effort,

\footnotetext{
${ }_{6}^{6}$ Below we will prove it wrong.

${ }^{7}$ It would be more convenient to verify our deductions with physical examples, since physics is one of the best developed theoretical and experimental sciences. If our logical deductions contradict physics, it will indicate an error in the approach proposed.

8 A concrete and somewhat strange example is chosen, because for further analysis we need to include a whole range of different qualities (both physical and psychological) into the description of the phenomenon.

9 There are other object characteristics that behave in the same way as rigidity: force, energy, etc. As will be further shown, they are derived from mass, and hence, they are not
} properties. and not only regarding physical velocity $Q_{1}$, but also the rest of characteristics ${ }^{10}$

Therefore, intensity of a property (quality expressedness) and its stability (the quantity of a given quality) - are two different notions ${ }^{11}$. It should be noted that property intensities are partial object characteristics, while rigidity (mass) - its integral characteristic. As related to that, we have to introduce new symbols: let us refer to the intensity of the $j$-th property as $V_{\mathrm{j}}$, and object rigidity - as $U_{\mathrm{H}}$.

Therefore, object definition in our case would be specified by the set $\left\{V_{1}, V_{2}, U_{\mathrm{H}}\right\}$, i.e. $\Omega=\Omega^{\prime} \rightarrow\left\{V_{1}, V_{2}, U_{\mathrm{H}}\right\}$, while object composition must comply with the rule:

$$
\Omega_{\Sigma}=\Omega+\Omega^{\prime} \rightarrow\left\{V_{1}, V_{2}, 2 U_{\mathrm{H}}\right\} .
$$

Evidently enough, vector representation of objects becomes impossible now, since the rule for object composition (2) does not conform to the rule of vector composition (1). To return to vector representation, let us associate vector $\vec{U}_{\mathrm{j}}$ to each $\mathrm{j}$-th quality in a certain space. It is evident that this vector has a certain correlation to intensity of $V_{\mathrm{j}}$ property. The space, in which such a representation is realized, will henceforth be called semantic space.

The situation described is represented in Figure 1. Since we have only three object characteristics, the semantic space will be three-dimensional. Here axis $e_{1}$ is correlated to the first characteristic, $e_{2}-$ with the second one and $e_{H}$ - with $U_{H}$, and taken together, they determine the Cartesian coordinates. Vector $\vec{U}_{\Sigma}=\vec{U}^{\prime}+\vec{U}$ has semantic coordinates: $\vec{U}_{\Sigma}=\left\{U_{\Sigma 1}, U_{\Sigma 2}, 2 U_{H}\right\}$. Let us designate plane $\left(\vec{e}_{\mathrm{j}} \times \vec{e}_{H}\right)$ through $\Upsilon_{\mathrm{j}}$ - the plane of the $\mathrm{j}$-th property.

We see that coordinates $U_{\Sigma 1}$ and $U_{\Sigma 2}$ of vector $\vec{U}_{\Sigma}$ are twice as great as for vector $\vec{U}\left(U_{1}=U_{\Sigma 1} / 2\right.$ and $\left.U_{2}=U_{\Sigma 2} / 2\right)$. However, $\varphi_{1}$ angle of incli-

\footnotetext{
${ }^{10}$ To have twice as many gulps, to stain twice as much clothes, etc., than in the case of one cup.

${ }^{11}$ For example, Например, work efficiency, on the one hand, can be characterized as intensity of performing a certain task per time unit. On the other hand, it could be described as a length of performing the task with a given intensity. Essentially, any psychological characteristic can be similarly described: sociability, extroversion, anxiety etc. It is known in the sphere of sociology that social tendencies, attitudes, prejudices and the like, have a great rigidity value. Yet in different historical periods, they can develop with various intensity values: both high and low.
} 


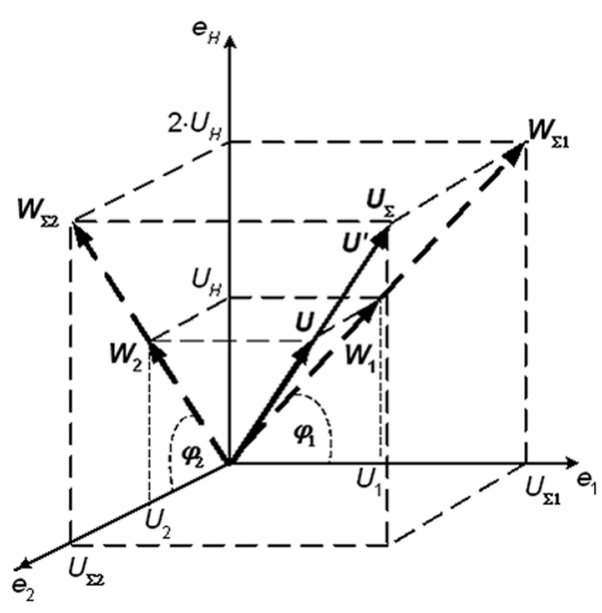

Figure 1. Construction of Semantic Coordinates

nation between projections of vectors $\vec{U}_{\Sigma}$ and $\vec{U}_{\Sigma}$ onto plane $\Upsilon_{1}\left(\vec{W}_{1}\right.$ and $\vec{W}_{\Sigma 1}$ correspondingly) toward $e_{1}$ axis has remained intact. A similar statement can be repeated concerning angle $\varphi_{2}$, that characterizes the slope of $\vec{W}_{2}$ and $\vec{W}_{\Sigma_{2}}$ to $e_{2}$ axis onto the plane of the second characteristic $\left(\Upsilon_{2}\right)$. As noted above, the object "doubled", his properties do not change, unlike the rigidity. That is why, the best "candidates" for the description of intensity of $V_{\mathrm{j}}$ properties are $\varphi_{\mathrm{j}}$ angles (or their derivatives) laid off on the planes of the j-th property. Therefore, characteristics in the semantic space would be specified on the flat continuum, and not the linear continuum, since it is impossible to specify an angle on the line.

Now let us verify, how adequate to Reality we are in proposing such a way of phenomena description, and what effect it brings about. Besides, it would be important to find out the sense of semantic coordinates of $U_{j}$.

Since $\varphi_{\mathrm{j}}=\arccos \left(U_{\mathrm{j}} / W_{\mathrm{j}}\right)$, it would be convenient to juxtapose the cosines of angles $\varphi_{j}(j=1,2, \ldots, n)$ to the corresponding property intensities $V_{\mathrm{j}}$. therefore, property intensities would be best put as follows:

$$
V_{\mathrm{j}}=C_{\mathrm{j}} \cdot \cos \varphi_{\mathrm{j}}=C_{\mathrm{j}} \cdot\left(U_{\mathrm{j}} / W_{\mathrm{j}}\right)=C_{\mathrm{j}} \cdot\left(U_{j} / \sqrt{U_{j}^{2}+\left(U_{\mathrm{H}}\right)^{2}}\right),
$$

where $C_{\mathrm{j}}$ - constants or scaling ratios that depend on the system of property units ( $\mathrm{j}-\mathrm{is}$ a property index).
Therefore, true characteristics will always be quantitatively restricted in the mental map, since $|\cos \varphi| \leq 1$. That is exactly why the scales for informant testing in psychology and sociology could be given by finite line segments with marked limits $C_{j}$. It should be noted that measurement of psychological characteristics is always realized in the restricted scales that have limiting values (sthenes, T-points etc.), and it would immediately cause a whole number of paradoxes. R. Ackoff (Ackoff, 1999), for example, proposes an interesting approach to forming psychologically compatible professional teams. He recognizes several factors of personal adaptability (internalization-externalization and subjectoversion-objectoversion), in which terms he describes separate individuals as vectors in a space of adaptability factors (with the limiting values $C_{\mathrm{j}}= \pm 1$ ). However, the description of characteristics of the whole group in that space often proves impossible, since summing of separate individual factor intensities causes violation of limiting values for those characteristics. Assuming that our object conceptualization is adequate, there should never occur such contradictions in the process of formalization.

Let us now test the correctness of our statements with the example of changing intensity of a certain quality, that is, deduce the general rule for intensity composition. Since $V_{\mathrm{j}}=C_{\mathrm{j}} \cdot \cos \varphi_{\mathrm{j}}$, then the angular coordinate for $U_{\mathrm{H}}$ in plane $\Upsilon_{j}$ would be determined as follows: $V_{\mathrm{Hj}}=C_{\mathrm{j}} \cdot \sin \varphi_{\mathrm{j}}$ and, therefore, we can put down the identity: $C_{\mathrm{j}}^{2}=V_{\mathrm{Hj}}^{2}+V_{\mathrm{j}}^{2}$ or $V_{\mathrm{Hi}}^{2}=C_{\mathrm{j}}^{2}-V_{\mathrm{j}}^{2}$.

By dividing the latter identity by $V_{\mathrm{Hj}}^{2}$ we receive:

$$
1=\left(C_{\mathrm{j}} / V_{\mathrm{Hj}}\right)^{2}-\left(V_{\mathrm{j}} / V_{\mathrm{Hj}}\right)^{2} .
$$

To simplify further calculations, let us resort to a formal technique and put the last identity as:

$$
1=\operatorname{ch}^{2} \theta_{\mathrm{j}}-\operatorname{sh}^{2} \theta_{\mathrm{j}}
$$

where ch $\theta_{\mathrm{j}}=C_{\mathrm{j}} / V_{\mathrm{Hj}}$ and sh $\theta_{\mathrm{j}}=V_{\mathrm{j}} / V_{\mathrm{Hj}}$ are hyperbolic cosine and sine respectively ${ }^{12}$.

Since th $\theta_{\mathrm{j}}=\operatorname{sh} \theta_{\mathrm{j}} / \operatorname{ch} \theta_{\mathrm{j}}=\left(V_{\mathrm{j}} / V_{\mathrm{Hj}}\right) /\left(C_{\mathrm{j}} / V_{\mathrm{Hj}}\right)=V_{\mathrm{j}} / C_{\mathrm{j}}$, then the rule for the composition of two intensities of the $\mathrm{j}$-th property $\left(V_{\mathrm{j}_{1}}\right.$ and

12 Whereas trigonometric are defined for segments functions in a circle, hyperbolic functions would be defined in hyperbola. The difference between trigonometric equations and hyperbolic ones can only be traced in signs (Bronstein, and Semendyayev, 1980, p. 279-282). 
$\mathrm{V}_{\mathrm{j}_{2}}$ ) would be determined by the formula for the tangent of two angles summed. Indeed, having represented a hyperbolic tangent as a sum of two angles, we would have:

$$
V_{\mathrm{j}} / C_{\mathrm{j}}=\operatorname{th} \theta_{\mathrm{j}}=\operatorname{th}\left(\theta_{\mathrm{j}_{1}}+\theta_{\mathrm{j}_{2}}\right)=\frac{\operatorname{th} \theta_{\mathrm{j}_{1}}+\text { th } \theta_{\mathrm{j}_{2}}}{1+\operatorname{th} \theta_{\mathrm{j}_{1}} \cdot \operatorname{th} \theta_{\mathrm{j}_{2}}}=\frac{\left(V_{\mathrm{j}_{1}} / C_{\mathrm{j}}\right)+\left(V_{\mathrm{j}_{2}} / C_{\mathrm{j}}\right)}{1+V_{\mathrm{j}_{1}} \cdot V_{\mathrm{j}_{2}} / C_{\mathrm{j}}^{2}}
$$

Therefore, canceling $C_{\mathrm{j}}$, we receive:

$$
V_{\mathrm{j}}=\frac{V_{\mathrm{j}_{1}}+V_{\mathrm{j}_{2}}}{1+V_{\mathrm{j}_{1}} \cdot V_{\mathrm{j}_{2}} / C_{\mathrm{j}}^{2}} \text {. }
$$

This general rule of property composition (4) completely coincides with the rule of velocity composition in relativist mechanics and is realized in Minkovsky space. At $V_{\mathrm{j}}<<C_{\mathrm{j}}$ Eq. (4) assumes a classical form: $V_{\mathrm{j}}=V_{\mathrm{j}_{1}}+V_{\mathrm{j}_{2}}$. However, while most problems in physics are solved with the use of a classical setting, in sociology and psychology consideration of non-linearity is a must, due to a lower rigidity of the properties studied. Even an ordinary calculation of mean values can lead to grave errors. It is easy to test that even the sum of limiting values $C_{j}$ still yields a limiting value:

$$
\frac{C_{\mathrm{j}}+C_{\mathrm{j}}}{1+C_{\mathrm{j}} \cdot C_{\mathrm{j}} / C_{\mathrm{j}}^{2}}=\frac{2 C_{\mathrm{j}}}{2}=C_{\mathrm{j}}
$$

Evidently, with such an approach to object description, the contradictions similar to those discussed in R. Ackoff's conception, are impossible.

Thus, the semiotic way of object description that we have chosen (object representation in the mental map) results in complete compliance with physical ideas of Reality. No doubt, the correlations received are objective and universal for all representatives of Homo Sapiens. However, they concern the mental way of Reality representation, rather than Reality itself.

As an example, we can draw some parallels to paradoxes in cartography, when practical necessity makes us map the Earth in flat Euclidean projections - topographical maps. As far as our research stayed within the boundaries of small areas, where the laws of our perception developed, there have been no paradoxes. But it is evidently impossible to construct an overall "flat" picture of the whole world without distortions that increase with approximation to specific points (normally, the Earth poles). It is reasonable that topological and metrical distortions can be expressed as a precise "objective law", although it is more closely related to the way of reality conceptualization, than "objective reality" itself.

Thus, restriction by limiting values ${ }^{13}\left( \pm C_{j}\right)$, of all properties within the limits of their intensity is a mere effect of a definite way of object representation on the mental map, which makes it finite and "visible" for the subject. The cost of it is a non-linear character of property composition

A good example for that may be found in expert evaluation of athletic achievements etc. Our mentality subconsciously perceives this as a natural increase in difficulty, the closer we are to perfection: every next step is harder than the previous one, while the difference in achievements is hardly noticeable. Indeed, let ideal $C$ equal one, and we are making the difference between the intensities $(0.2$ and 0.1$)$ of a certain property of two objects. The difference would run: $(0.2-0.1) /\left(1-0.2 \cdot 0.1 / 1^{2}\right) \approx 0.1$ as proper in the classical theory. However, for the expressed properties of 0.9 and 0.8 the actual difference would be greater: $(0.9-0.8)$ / $\left(1-0.9 \cdot 0.8 / 1^{2}\right) \approx 0.357$. Therefore modification inputs ${ }^{14}$ related to the intensity of a property (for example, athletic result, personal characteristic or quality of goods) would run much larger in the second case, although mental differences in perception of property intensities in the first and second cases are the same $(0.2-0.1=0.9-0.8)$.

Let us now clarify the sense of $V_{\mathrm{Hj}}$. Basing on Eq. (3) and Eq. (4), it is easy to deduce a composition formula for the value $V_{\mathrm{Hj}}$ introduced:

$$
\begin{gathered}
V_{\mathrm{H}_{\mathrm{j}}}^{2}=C_{\mathrm{j}}^{2}-V_{\mathrm{j}}^{2}=C_{\mathrm{j}}^{2}-\left(\frac{V_{\mathrm{j}_{1}}+V_{\mathrm{j}_{2}}}{1+V_{\mathrm{j}_{1}} \cdot V_{\mathrm{j}_{2}} / C_{\mathrm{j}}^{2}}\right)^{2}= \\
=\frac{\left(C_{\mathrm{j}}-V_{\mathrm{j}_{1}}\right)^{2}-V_{\mathrm{j}_{2}}^{2}\left(1-V_{\mathrm{j}_{1}}^{2} / C_{\mathrm{j}}^{2}\right)}{\left(1+V_{\mathrm{j}_{1}} \cdot V_{\mathrm{j}_{2}} / C_{\mathrm{j}}^{2}\right)^{2}}=\frac{V_{\mathrm{H}_{1}}^{2} \cdot V_{\mathrm{Hj}_{2}}^{2}}{C_{\mathrm{j}}^{2}\left(1+V_{\mathrm{j}_{1}} \cdot V_{\mathrm{j}_{2}} / C_{\mathrm{j}}^{2}\right)^{2}} \\
V_{\mathrm{H}_{\mathrm{j}}}=\frac{V_{\mathrm{Hj}_{1}} \cdot V_{\mathrm{Hj}_{2}}}{C_{\mathrm{j}}\left(1+V_{\mathrm{j}_{1}} \cdot V_{\mathrm{j}_{2}} / C_{\mathrm{j}}^{2}\right)}=\frac{V_{\mathrm{Hj}_{1}} \cdot \sqrt{1-V_{\mathrm{j}_{2}}^{2} / C_{\mathrm{j}}^{2}}}{C_{\mathrm{j}}\left(1+V_{\mathrm{j}_{1}} \cdot V_{\mathrm{j}_{2}} / C_{\mathrm{j}}^{2}\right)} .
\end{gathered}
$$

\footnotetext{
${ }_{13}$ This explains, in particular, the reason for physical limitation of velocities by velocity of light, as well as strange paradoxes of the special relativity theory. Is should be stressed, one more time, that the reason here is not physical, but psychological.

${ }^{14}$ Inputs are proportional to energy (see below)
} 
Every individual possesses and can be described through a specific set of qualities and their intensities: strength, balance, mobility of neural processes, temperament, character etc. In fact he represents a certain concrete reference frame, through which "external" processes are perceived and estimated. Obviously enough, all outside phenomena would be perceived differently through different reference frames, due to individual differences ${ }^{15}$.

Let us take a situation, where two individuals are perceiving a certain process defined by the sequence of the changing parameter $x:\left\{x_{1}, x_{2}, \ldots\right.$, $\left.x_{\mathrm{T}}, \ldots\right\}$, and characterized by specific intensity of a certain $V_{\mathrm{j}}$ property. In psychology, for example, a lot of personality traits are directly determined by the frequency of manifestation of certain qualities, that is why $V_{\mathrm{j}}$ refer to such attributes as aggressiveness, anxiety, falsity etc. Their intensity is the higher, the more often relevant behavior repeats within a time unit ${ }^{16}$. The simplest example of $V_{\mathrm{j}}$ property in physics is the speed of constant motion of a body, $\boldsymbol{x}$ parameter in this case being the path traveled by the body.

It is reasonable that all $V_{\mathrm{j}_{1}}$ in one's "personal" reference system that define the first individual, be equated to zero (for him they are reference points to prescribe his reference system), while the second one $\left(V_{\mathrm{j}_{2}}\right)$ be given the intensity value of a studied characteristic equaling the difference $\Delta V_{\mathrm{j}}=V_{\mathrm{j}_{1}}-V_{\mathrm{j}_{1}}$.

Let us introduce the concept of "absolute" time $\mathrm{T}$, as a certain index ${ }^{17}$ to be "ascribed" by the subject in "his" reference system to all sequential forms of current world processes:

$$
x_{1} \stackrel{\mathrm{S}}{\longrightarrow} x_{2} \stackrel{\mathrm{s}}{\longrightarrow} \cdots \stackrel{\mathrm{s}}{\longrightarrow} x_{\mathrm{T}} \text {. }
$$

Here $\mathbf{S}$ is the operator that determines the sequence of forms (meanings) of a certain process. Since the dynamism ${ }^{18}$ (rate) of the $x$ alteration must depend on rigidity or inertness of a specific quality, we may try to relate its variability to $V_{\mathrm{H}_{j}}$, because this value concerns a concrete quality

${ }_{15}$ Depending on how well our own qualities are expressed (anxiety, sociability, work efficiency, etc.) we will estimate them in other people. For example, Russians consider imperturbability a national characteristic of Finns, because even the most "excitable" Finn seems to be a phlegmatic, compared with a Russian.

${ }^{16}$ For example, if $V_{\mathrm{j}}$ is work efficiency, then $x$ - is amount of work performed within time $t$.

17 Index, enumerating the sequence of forms: $x_{1}, x_{2}, \ldots, x_{\mathrm{T}}$, of a current process.

${ }^{18}$ Degree of a property variability or rigidity. (j) and is derived from the $U_{\mathrm{H}}$ index that characterizes object rigidity. Let us define $V_{\mathrm{H}_{\mathrm{j} 1}}$ as the "speed of indexation" of the $\boldsymbol{x}$ parameter («speed of time flow» in subjective perception) or rigidity of the $j$-th property in the first system of reference. Therefore, subjective time of the studied process realization (from 0 to $\mathrm{T}$ ) would be defined by the first individual as $t=V_{\mathrm{H}_{\mathrm{j} 1}} \cdot \mathrm{T}$, and by the second individual - as $t^{\prime}=V_{\mathrm{H}_{\mathrm{j}}} \cdot \mathrm{T}$. This would mean that degrees of dynamism (or progress speeds) of processes are differently perceived in different systems of reference.

Let us test our assumption. Employing transformation (5), we obtain:

$$
t^{\prime}=T \cdot V_{\mathrm{H}_{\mathrm{j} 2}}=\frac{T \cdot V_{\mathrm{H}_{\mathrm{j} 1}} \cdot \sqrt{1-\left(\Delta V_{\mathrm{j}}\right)^{2} / C^{2}}}{1+V_{\mathrm{j}} \cdot \Delta V_{\mathrm{j}} / C^{2}}=\frac{t \cdot \sqrt{1-\left(\Delta V_{\mathrm{j}}\right)^{2} / C^{2}}}{1+V_{\mathrm{j}} \cdot \Delta V_{\mathrm{j}} / C^{2}} .
$$

Therefore,

$$
t=\frac{t^{\prime}+\left(\Delta V_{\mathrm{j}} / C^{2}\right) V_{\mathrm{j}} \cdot t^{\prime}}{\sqrt{1-\left(\Delta V_{\mathrm{j}}\right)^{2} / C^{2}}} .
$$

If property $V$ is speed, then $V \cdot t^{\prime}=x^{\prime}$ and

$$
t=\frac{t^{\prime}+\left(\Delta V / C^{2}\right) x^{\prime}}{\sqrt{1-(\Delta V)^{2} / C^{2}}}
$$

specifies time transformation for physical processes at the transition into another inertial system, which coincides with the well-known Lorenz transformations and supports our assumption concerning $V_{\mathrm{H}}$, as a characteristic determining subjective time flow velocity. It is just as easy to obtain transformations for coordinates, relevant to the special relativity theory. It should be noted that "time slow-down" effects can be observed not only in physics in case of extremely high speeds, but in psychology as well due to the extreme changes in individual's psychical characteristics ${ }^{19}$. Many people have personally experienced changes in time flow velocity under stress conditions.

19 There is the so-called "hypermnesia of the drowning" (official name) described in psychology. It is manifested in people in times of mortal danger. People who have come through the danger of inevitable death say that the whole of their life ran before them in distinct detail, beginning from the last moments to pictures of early childhood. Such kind of hypermnesia was experienced and described by the British hydrographer admiral Francis Beaufort, who was saved after the shipwreck. Similar effects have been traced in lethargic sleep. The changes in the time scale (both toward acceleration and deceleration) could be suggested through hypnosis (Grimack, 1978). 
We can further try testing our deduction for consistency with physics. Let us, for example, deduce the Doppler effect equation. For a wave process with $\tau$ period we have:

$$
\tau^{\prime}=V_{\mathrm{H}}^{\prime} \cdot \Delta T=\frac{\Delta T \cdot V_{\mathrm{H}} \cdot \sqrt{1-(\Delta V)^{2} / C^{2}}}{1+\Delta \vec{V} \cdot \vec{C} / C^{2}}=\frac{\tau \cdot \sqrt{1-(\Delta V)^{2} / C^{2}}}{1+(\Delta V / C) \cos \theta} .
$$

Since $\tau=1 / \omega$, then

$$
\omega^{\prime}=\frac{\omega \cdot \sqrt{1-(\Delta V)^{2} / C^{2}}}{1 \pm(\Delta V / C) \cos \theta}
$$

Thus, the rules of speed (4), time (6), coordinates transformation, Doppler effect (7) (Landau, and Lifschitz 1973, p. 154) are essentially the result of a specific way of Reality conceptualization in our mentality. Beyond the conditions of natural biological adaptation and in proximity to "the ideal $C$ " (for $\varphi \approx 0$ ) it effects in paradoxical Reality perception Here all the relativist effects are essentially psychological (mental), and not physical.

Thus, we have demonstrated that adequate object description through its properties in any theory is only possible in the semantic space, and it obligatory requires not only measurements of intensities of characteristic, but their rigidities as well. The reason for it is that characteristics are represented in the flat continuum $\left(\vec{e}_{\mathrm{j}} \times \vec{e}_{\mathrm{H}}\right)$, and not in the one-dimensional continuum $\left(\vec{e}_{\mathrm{j}}\right)$, as has been shown above.

Let us now gain calculation formula for rigidity $U_{\mathrm{H}}$ and semantic coordinates of objects $U_{\mathrm{j}}$ in a general case. It issues from the Formula 1 (see also Figure1), that

$$
\operatorname{ctg} \varphi_{\mathrm{j}}=U_{\mathrm{j}} / U_{\mathrm{H}}=v_{\mathrm{j}} / v_{\mathrm{Hj}}=u_{\mathrm{j}} / u_{\mathrm{H}},
$$

where $u_{\mathrm{j}}$ and $u_{\mathrm{H}}$ are normalized semantic coordinates, and $v_{\mathrm{H}}$ are normalized property intensities ${ }^{20}$ :

$$
\begin{gathered}
\vec{u}=\vec{U} /|\vec{U}|, \\
v_{\mathrm{Hj}}=V_{\mathrm{Hj}} / C_{\mathrm{j}}=\sin \varphi_{\mathrm{j}}, \\
v_{\mathrm{j}}=V_{\mathrm{j}} / C_{\mathrm{j}}=\cos \varphi_{\mathrm{j}} .
\end{gathered}
$$

$\overline{20}$ It is more convenient to use property scales with limiting values \pm 1 in informant testing.
It issues from Eq. (8) that:

$$
U_{\mathrm{j}}=U_{\mathrm{H}} \cdot\left(v_{\mathrm{j}} / v_{\mathrm{Hj}}\right) .
$$

Since,

$$
\begin{aligned}
\overrightarrow{\mathrm{U}}^{2} & =\overrightarrow{\mathrm{U}}^{\prime 2}+\overrightarrow{\mathrm{U}}_{\mathrm{H}}^{2}=\sum_{\mathrm{j}=1}^{\mathrm{n}} U_{\mathrm{j}}^{\prime 2}+U_{\mathrm{H}}^{2}=\sum_{\mathrm{j}=1}^{\mathrm{n}} U_{\mathrm{H}}^{2} \cdot \frac{v_{\mathrm{j}}^{2}}{v_{\mathrm{Hj}}^{2}}+U_{\mathrm{H}}^{2}= \\
& =U_{\mathrm{H}}^{2} \cdot\left[\left(\sum_{\mathrm{j}=1}^{\mathrm{n}} \frac{v_{\mathrm{i}}^{2}}{v_{\mathrm{Hj}}^{2}}\right)+1\right]=U_{\mathrm{H}}^{2} \cdot\left[\left(\sum_{\mathrm{j}=1}^{\mathrm{n}} \frac{v_{\mathrm{j}}^{2}}{1-v_{\mathrm{j}}^{2}}\right)+1\right],
\end{aligned}
$$

then

$$
U_{\mathrm{H}}=\frac{|\overrightarrow{\mathrm{U}}|}{\sqrt{\sum_{\mathrm{j}=1}^{\mathrm{n}}\left(v_{\mathrm{i}}^{2} /\left(1-v_{\mathrm{j}}^{2}\right)\right)+1}} .
$$

Here

$$
\vec{U}^{\prime}=\left\{U_{1}, U_{2}, \ldots, U_{\mathrm{n}}, 0\right\} ; \vec{U}_{\mathrm{H}}=\left\{0,0, \ldots, U_{\mathrm{H}}\right\} .
$$

It issues from Eq. (10) and Eq. (9) that:

$$
\begin{gathered}
u_{\mathrm{H}}=\frac{1}{\sqrt{\sum_{\mathrm{j}=1}^{\mathrm{n}}\left(v_{\mathrm{j}}^{2} /\left(1-v_{\mathrm{j}}^{2}\right)\right)+1}} ; \\
u_{\mathrm{j}}=u_{\mathrm{H}} \cdot \frac{v_{\mathrm{j}}}{v_{\mathrm{Hj}}}=u_{\mathrm{H}} \cdot \frac{v_{\mathrm{j}}}{\sqrt{1-v_{\mathrm{j}}^{2}}}=u_{\mathrm{H}} \cdot \frac{V_{\mathrm{j}} / C_{\mathrm{j}}}{\sqrt{1-V_{\mathrm{j}}^{2} / C_{\mathrm{j}}^{2}}} ;
\end{gathered}
$$

Eq. (11) is used for calculation of normed minimal object rigidity as a whole, while Eq. (12) - for calculation of normed semantic coordinates. Then absolute semantic coordinates $U_{j}$ could be obtained through the formula:

$$
U_{\mathrm{j}}=\frac{V_{\mathrm{j}} \cdot\left(u_{\mathrm{H}} \cdot|\overrightarrow{\mathrm{U}}| / C_{\mathrm{j}}\right)}{\sqrt{1-V_{\mathrm{j}}^{2} / C_{\mathrm{j}}^{2}}}=\frac{V_{\mathrm{j}} \cdot m_{0 \mathrm{j}}}{\sqrt{1-V_{\mathrm{j}}^{2} / C_{\mathrm{j}}^{2}}}=m_{\mathrm{j}} V_{\mathrm{j}} .
$$

In physics the last equation could be put down as follows:

$$
P_{\mathrm{j}}=m_{\mathrm{j}} \cdot V_{\mathrm{j}},
$$

where $P_{\mathrm{j}}$ is impulse, $V_{\mathrm{j}}$ - velocity, and $m_{\mathrm{j}}$ - object mass. 
In Eq. (13) $m_{0 j}$ is «zero» rigidity ${ }^{21}$, and $m_{j}$ - full rigidity of the $j$-th characteristic: $m_{0 \mathrm{j}}=u_{\mathrm{H}} \cdot|\vec{U}| / \mathrm{C}_{\mathrm{j}}$,

$$
m_{\mathrm{j}}=\frac{m_{0 \mathrm{j}}}{\sqrt{1-V_{\mathrm{j}}^{2} / C_{\mathrm{j}}^{2}}} \cdot
$$

It is clear from Eq. (15) that full rigidity of a characteristic $m_{\mathrm{j}}$ grows in a non-linear fashion with the rising intensity of property $V_{\mathrm{j}}$. In fact, it means that an absolute limit of any property is a thing unattainable. It should be noted that Eq. (14) actually coincides with the expression for impulse in relativist mechanics ${ }^{22}$.

Therefore, semantic coordinates that give the "adequate/ complete" semantic description of an object on the mental map, are similar to what physicists call impulse representation. It is in the semantic representation that semantic description of a closed system of objects is retained in any instances of its transformation, which, by the way, reveals the sense of the impulse itself $\mathrm{f}^{23}$. Moreover, it can be shown that the laws of "sense conservation" become equivalent to laws of energy and impulse conservation in the relativist theory. It means a basic possibility of applying laws of conservation in the humanities, which makes their prediction potential equivalent to that of the natural sciences ${ }^{24}$. Apparently, these laws can be used only in case of adequate semantic representation of the studied

\footnotetext{
${ }^{21}$ Rigidity at zero property intensity
}

22 One can conduct theoretical testing of adequacy of semantic representation of objects and phenomena only in physics, since in the humanities (including psychology) rigidity of the characteristics studied is not only disregarded, but there is no such a concept as "rigidity of a characteristic" elaborated within their theories.

${ }^{23}$ Using impulses, physicists implicitly transfer to a semantic space, which is the only sphere where conservation laws can be observed. Thus for calculation of body interactions, they first transfer to impulse setting, perform their calculations there, and then "return" back to properties.

${ }^{24}$ Semantic laws of conservation remind of accounting of costs of funds available. If they are completely listed, then their respective sums, debit and credit, are retained. In this sense, "accounting" of semantic units, describing phenomena, is more effectively organized in physical theories than in the humanities. However, there is no basic difference between them in this sense. All formal theories must be equivalent from the point of view of prediction accuracy. The only fact that makes them different is different sides of Reality that they study, using their specific descriptors for the purpose. For example, we can study coffee from the point of view of physics, chemistry, gastronomy, etc.

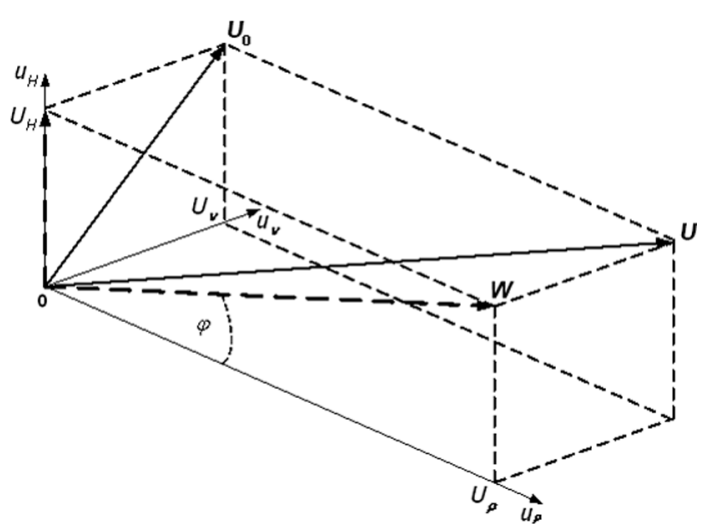

Figure 2. Semantic Invariants

phenomena in theory, which has been discussed above. It does not only require obligatory measurement of object properties important for a researcher, with their intensities, but their rigidities as well.

Let us obtain the second fundamental law of conservation - the law of energy conservation and the well-known correlation for energy and mass $E=m \cdot C^{2}$. We will make use of only psychosemiotic principles of phenomena description.

It would be necessary to discuss a classical case, when it is possible to change one $\rho$ property of a certain object ${ }^{25}$, defined by vector $\vec{U}_{0}=$ (with the initial $\left.U_{\rho}\right)$, values of other semantic coordinates $\left(U_{v}\right)$ remaining fixed. Let us exemplify it. Imagine that we have a box. We can change the length, height or width of the box within a certain admissible range, and it will still belong to the category of three-dimensional bodies. Yet nullification of one of these values immediately transfers it into the category of flat figures. It should be noted that some object characteristics can be excluded from its definition as it is, and that is why they can change within any range (for example, color, location or speed of the box).

\footnotetext{
${ }^{25}$ In this sense an object can be an individual, a personality, character, team, product image, service etc., that is - anything that has a concrete definition, can be described through properties and is representable in the semantic space. It is also important that a semantic space can be stratified, and any quality can be of any dimensionality (for example, speed $\mathbf{V}=\left\{V_{\mathrm{x}}, V_{\mathrm{p}}, V_{z}\right\}$ ).
} 
The change in $U_{\rho}$ semantic coordinate of $\vec{U}_{0}$ object, from 0 to $U_{\rho}$ transforms the vector that determines an object, from state $\vec{U}_{0}$ to state $\vec{U}$. In this connection, projections of semantic $\vec{U}$ vector onto $e_{v}$ axis must remain unaltered under consistent changes of $U_{\rho}$, which is equivalent to the change in only one property $\rho$ of the object (See Fig. 2). The change in semantic coordinate $U_{\rho}$ of the object is only possible in case of increase of the length of semantic vector $\vec{U}$, that describes the object. Projection of $\vec{U}$ vector onto $\vec{e}_{v} \times \vec{e}_{\mathrm{H}}$ plain remains unchanged and equals $\vec{U}_{0}$, while projection $W_{\rho}$ of $U$ semantic vector onto $u_{\mathrm{H}} \times u_{\rho}$ plane steadily grows.

Let us express the above alterations in terms of properties:

$$
\Delta \vec{U}=\vec{U}-\vec{U}_{0}=\vec{W}_{\rho}-\vec{U}_{\mathrm{H}}=\vec{U}_{\rho},
$$

since $U_{0}-U, U_{\mathrm{H}}-W_{\rho}$ and $0-U_{\rho}$ segments are equal (see Fig. 2). Therefore, through introducing $\phi$ angle between vectors $\vec{U}_{\rho}$ and $\vec{W}_{\rho}$, we receive:

$$
\begin{gathered}
U_{\rho}^{2}=\left(\vec{W}_{\rho}-\vec{U}_{\mathrm{H}}\right)^{2}=\vec{W}_{\rho}^{2}-2 \cdot\left|\vec{W}_{\rho}\right| \cdot\left|\vec{U}_{\mathrm{H}}\right| \cos (\pi / 2-\varphi)+\vec{U}_{\mathrm{H}}^{2}= \\
=\left(U_{\mathrm{H}} / \sin \varphi\right)^{2}-2\left(U_{\mathrm{H}} / \sin \varphi\right) U_{\mathrm{H}} \sin \varphi+\vec{U}_{\mathrm{H}}^{2}=\left(U_{\mathrm{H}} / \sin \varphi\right)^{2}-\vec{U}_{\mathrm{H}}^{2}= \\
=\left(\frac{U_{\mathrm{H}}}{\sqrt{1-\cos ^{2} \varphi}}\right)^{2}-U_{\mathrm{H}}^{2}=\frac{1}{C^{2}}\left(\frac{\left(U_{\mathrm{H}} / C\right) C^{2}}{\sqrt{1-V^{2} / C^{2}}}\right)^{2}-\frac{1}{C^{2}}\left(\frac{U_{\mathrm{H}}}{C} C^{2}\right)^{2}= \\
=\left(\frac{U_{\mathrm{H}}}{\sqrt{1-\cos ^{2} \varphi}}\right)^{2}-U_{\mathrm{H}}^{2}==\frac{1}{C^{2}}\left(\frac{\left(U_{\mathrm{H}} / C\right) C^{2}}{\sqrt{1-V^{2} / C^{2}}}\right)^{2}-\frac{1}{C^{2}}\left(\frac{U_{\mathrm{H}}}{C} C^{2}\right)^{2}= \\
=\frac{1}{C^{2}}\left[\left(\frac{m_{0} C^{2}}{\sqrt{1-V^{2} / C^{2}}}\right)^{2}-\left(m_{0} C^{2}\right)^{2}\right]=\left(\frac{E^{2}}{C^{2}}-\frac{E_{0}^{2}}{C^{2}}\right)
\end{gathered}
$$

Where

$$
E=\frac{m_{0} C^{2}}{\sqrt{1-V^{2} / C^{2}}}=m C^{2} ; \quad E_{0}=m_{0} C^{2} .
$$

It would be worth reminding the fact that semantic coordinates $U_{\mathrm{j}}$ correspond to impulses $P_{\mathrm{j}}$ in physics (see Eq. (14)). Thus, we have obtained:

or

$$
U_{\rho}^{2}=P^{2}=\left(E^{2} / C^{2}-m_{0}^{2}-m_{0}^{2} C^{2}\right)
$$

$$
E^{2} / C^{2}=P^{2}+m_{0}^{2} C^{2} \text {. }
$$

Eq. (18) is known in physics as the relativist energy-impulse relation, while Eq. (16) reflect the relations between energy and mass (rigidity). It should be noted that, object mass is not an equipotent property (actually, it is no longer a property, but a summary characteristic of properties' rigidity), being defined through several independent values:

$$
m=\sqrt{U_{\mathrm{H}}^{2}+\sum_{\mathrm{i}} U_{\mathrm{i}}^{2}} \text {. }
$$

It issues from Eq. (19) that full mass (length of a semantic vector, determining an object), unlike properties, is not limited, that is why all the values depending on it, are not properties, either (for example, energy, temperature ${ }^{26}$, etc.).

Therefore, laws of impulse and energy conservation are adequate to conservation of "semantic definition" of an object, and, in their universal form, they are common to all sciences.

To make use of this potential in psychology and sociology, it is enough to transfer from object and phenomena representation in a space of characteristics to semantic representation through Equations (11), (12), (13), (15). For example, when an integrated description of a group of people is performed in the above mentioned theory by R. Ackoff, it is not the intensities of separate members' personal qualities $V_{\mathrm{j}}$ that are added together, but their $U_{j}$ semantic coordinates. Then, taking into account total summary rigidity of a group, one can determine intensities of group characteristics, the group to perceived as an integral whole ${ }^{27}$.

Since for the vector sum (concepts of objects and individuals) the following well-known relation holds true in the semantic space:

$$
\left|\vec{U}_{1}+\vec{U}_{2}\right| \leq\left|\vec{U}_{1}\right|+\left|\vec{U}_{2}\right|
$$

\footnotetext{
${ }^{26}$ In biology temperature can be used not as a physical exponent of molecule average kinetic energy, but as a physiological exponent o fan organism's health, and therefore it would have limited values correlated with vital physiological processes, and can be regarded as a property.

${ }^{27}$ The same is performed by physicists when calculating velocity of interacting bodies: first they turn to impulse representation, and having performed the necessary calculations, they get back to velocities. It is impossible to predict the effects of two bodies interaction, without considering mass (rigidity). For example, a change in body velocities (property intensities) after the collision of two iron balls, and an iron and a cork ball, would be different, other conditions equal. Yet, in principle, psychologists do not trace rigidities of characteristics studied, that is why valid predictions could not be expected in their theories.
} 
there arises an illusion of energy deficit. It is obvious that the summary "length" of vector-objects does not disappear, since mutual compensation works, instead of disappearance of semiotic units ${ }^{28}$. That is why the "vector" semantic law of conservation ("impulse" law) is by no means sufficient to describe changes in the system. Law of energy conservation serves as a natural supplement to it.

It should be noted that any particle $\vec{u}^{(+)}=\left\{u_{1}, u_{2}, \ldots, u_{\mathrm{n}}, u_{\mathrm{H}}\right\}$ described in the semantic space, could be contrasted with an anti-particle $\vec{u}^{(-)}=\left\{-u_{1},-u_{2}, \ldots,-u_{\mathrm{n}},-u_{\mathrm{H}}\right\}$ inverted in directions of all properties and time $^{29}$ (since the "time arrow"s defined by the $u_{\mathrm{H}}$ sign: $t=V_{\mathrm{H}} \cdot \mathrm{T}$ ). Time inversion actually means a change in particle direction under forces, which is equivalent to a change in charge sign. Besides, if we were to admit the Great Explosion theory and creation of the Universe out of vacuum, then the sum of all semantic coordinates in it must equal zero. It is only possible in the case of an anti-world presumably existing, with a negative $u_{\mathrm{H}}$. This anti-world has been at a time variance with our Universe since its very emerging period ${ }^{30}$.

Thus, an object adequately defined in any theory, including the humanities, the universal laws of conservation tend to function, by analogy with those applied in the natural sciences. Then, the theoretical potential of the humanities is equivalent to that of natural sciences, and possesses no actual difference.

\section{Testing of Theoretical Deductions}

The reason we have chosen a psychophysical example for demonstration of our approach opportunities is not that we are interested in this very problem. We wanted to deliberately avoid our own experimental material ${ }^{31}$, since it could cause additional doubt in the reader (in any

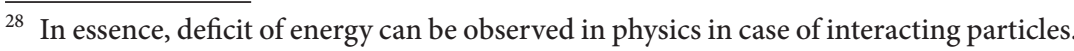
It is related to the transformation of part of kinetic energy into bonding energy.

29 The CPT theorem in physics demonstrates the symmetry on natural laws, in case of simultaneous realization of space inversion $(\mathrm{P})$, time reversal $(\mathrm{T})$ and charge coupling (C) - the substitution of particles for the respective anti-particles.

${ }^{30}$ Which, in terms of this theory, explains why the Universe did not annihilate itself directly after its emergence.

31 Although we have conducted such studies in the spheres of social, political, organiza-
} tional, gender psychology and psychology of personality. case, such material would require independent testing). The sphere of psychophysics was preferred only because it contains quite precise measurements tested by a number of independent researchers. Further, we will show the fact that semantic approach makes it possible to theoretically obtain all the points on the loudness curve, including those that do not conform to the S. Stevens formula.

Relativist correlations in the sphere of psychology could be tested on psychophysical material, since it is the only area, where psychologists take quite accurate measurements.

It is obviously impossible to provide the acceptable level of signal discrimination within one range in most sensor systems ${ }^{32}$. This is related to the phenomenon of sensor system adaptation to irritant average level, which is widely known in psychophysics. For example, when we leave a semi-dark room for bright sunshine, we are "blinded" for some time, until the sensor system "switches" to the new range. Eye sensitivity is known to increase 200000 times at transition from bright light to darkness $^{33}$ (Louriah, 1975).

Let us demonstrate practical application of relativist scales in psychology, with the example of correlation between subjective loudness and sound intensity. At the outset, it would be worth reminding basic psychophysical foundations in this sphere, and then go on with calculations of our method. $(d B)$ :

It is conventional to define the intensity of sound irritant in decibels

$$
L=20 \cdot \lg \left(P / P_{0}\right),
$$

where $P_{0}=2 \cdot 10^{-5} \mathrm{nt} / \mathrm{m}^{2}$.

Intensity of $40 \mathrm{~dB}(L)$ signal at a frequency $f=1000 \mathrm{~Hz}$ is standardized in psychophysics as a subjective loudness $(N)$ of 1 sone or $\left(L_{\mathrm{r}}\right)$ loudness of 40 phons (at sound frequency of $1000 \mathrm{~Hz}$ the scale of sound pressure in $d B$ and loudness scale in phons coincide).

Measurements of loudness curve in psychophysics start from the reference level of $40 d B$ (1 sone), relative to which the sound intensity level is determined, the sound subjectively perceived as twice louder. Thus, a number of sound intensity values are successively determined. Each one of these values corresponds to the loudness level twice as great

\footnotetext{
${ }^{32}$ On the loudness scale, for example, it will make $130 \mathrm{~dB}$.

${ }^{33}$ It happens due to the change in the pupil and pigment moving in the eye retina.
} 


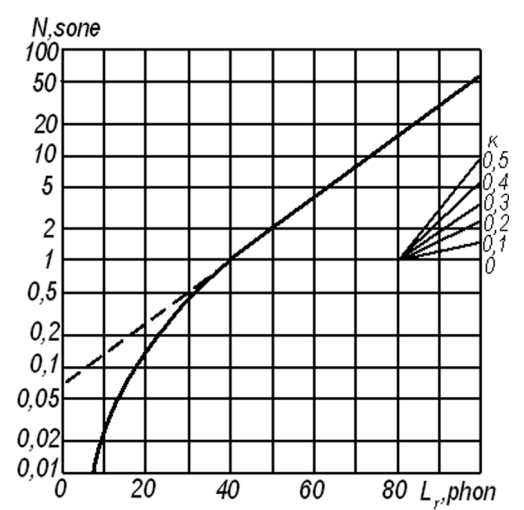

Figure 3. Relation between subjective loudness $N$ and loudness level $L_{r}$ (Zwicker, and Feldtkeller, 1967)

as the previous one, i.e. levels of $2,4,8,16,32,64$ and 128 sones. Then this series of tests is conducted in the reverse order from the basic value $(40 \mathrm{~dB})$, and the next number of sound intensity values are determined, each of those being twice as small as the previous one: $1 / 2,1 / 4,1 / 8,1 / 16$, $1 / 32$ sones (Zwicker, and Feldtkeller, 1967). Based on the points received through experiment, a researcher builds a corresponding interaction curve between a physical magnitude (level of acoustic pressure) and loudness, which is a perception indicator. This curve is depicted in Figure 3. It was S. Stevens who discovered similar regulations governing perception and irritation factors for a lot of sensations of different modalities. In the right part of Figure 3 there are several graphs of power function by S. Stevens, through which those curves are described:

$$
N=\operatorname{const}\left(P / P_{0}\right)^{2 \mathrm{k}} \text {. }
$$

In the area of levels higher than $40 \mathrm{~dB}$ this relation can approximately be reflected as a straight line (on a logarithmic scale). That is why an international agreement was reached on the point that relationship between loudness $N$ (in sones) and level $L_{\mathrm{r}}$ for a span of 40 to 100 phons should be expressed by the following formula:

$$
N=2^{\left(\frac{L_{\mathrm{r}}-40}{10}\right)} \text {. }
$$

Graph of the function defined through Eq. (20), is shown in Figure 3 as a dotted line. It is clear that only on the levels above 40 phons does this line coincide with the one experimentally obtained.

Now, using our method, let us theoretically calculate the relation between the subjective loudness and sound intensity. For average intensity of irritant of $L_{\mathrm{i}} d B$ at a frequency of $1000 \mathrm{~Hz}$ we will receive a correlation:

$$
\frac{P_{0} \cdot 10^{\frac{L}{20}}}{P_{0} \cdot 10^{\frac{L_{\mathrm{i}}}{20}}}=\frac{U_{\mathrm{H}} \cdot N / \sqrt{1-N^{2} / C^{2}}}{U_{\mathrm{H}} \cdot N_{\mathrm{i}} / \sqrt{1-N_{\mathrm{i}}^{2} / C^{2}}}=\frac{N \cdot \sqrt{C^{2}-N_{\mathrm{i}}^{2}}}{N_{0} \cdot \sqrt{C^{2}-N^{2}}},
$$

where $N_{\mathrm{i}}$ is the basic (average in the range) loudness level, corresponding to the stimulus intensity of $L_{\mathrm{i}} d B$, whereas $C$ is the limit loudness value in the $\mathrm{i}$-th range.

According to the experiment scheme, we suppose that $L_{0}=40 \mathrm{~dB}$ $(\mathrm{i}=0)$ and determine $L$, at which the subjective loudness is doubled. Stimulus intensities would be determined by the formula:

$$
\frac{P_{0} \cdot 10^{\frac{L}{20}}}{P_{0} \cdot 10^{\frac{L_{\mathrm{i}}}{20}}}=\frac{2 \cdot \sqrt{C^{2}-1^{2}}}{1 \cdot \sqrt{C^{2}-2^{2}}}
$$

Since the first doubling of loudness takes place at $L=50 \mathrm{~dB}$ ( 2 sones), therefore we can calculate $C=\sqrt{6} \approx 2.449$ sones.

Substituting $L_{1}=50$ into the left part of Eq. (22), and assuming that the corresponding subjective loudness value equals one (a new reference value of subjective loudness), let us calculate the second doubling of loudness (4 sones).

Loudness for the calculated value being $L=60 \mathrm{~dB}$, we again assume it for one, and, having substituted $L_{2}=60 \mathrm{~dB}$ into the left part of Eq. (22), we calculate the next value of $L_{3}=70 \mathrm{~dB}$ of doubling loudness (8 sones). Repeating that operation, we successively obtain the following values: $80 d B-16$ sones, $90 d B-32$ sones, $100 d B-64$ sones, $110 d B-128$ sones, $120 d B-256$ sones. Thus, we have theoretically obtained the values, which precisely agree with experimental studies and Stevens formula.

The loudness scheme (Figure 3 ) has a contrary flexure exactly at the point of $40 d B$, assumed by Stevens as a loudness unit. It is not Stevens' excellent intuition that made him select the level of $40 \mathrm{~dB}$. If Stevens had assumed the subjective loudness level at $50 \mathrm{~dB}$ for 1 sone, the graph 


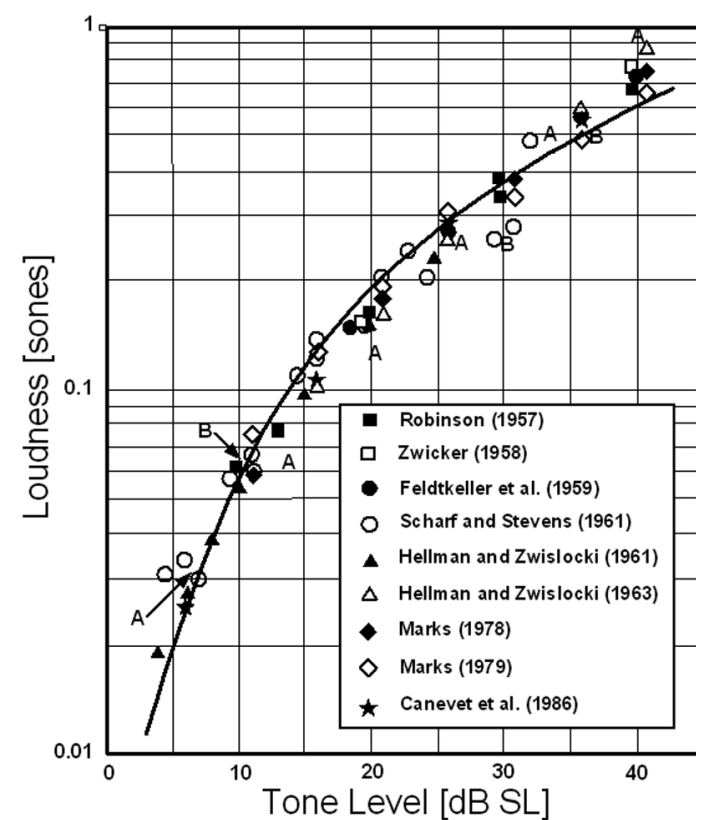

Figure 4. Loudness curve (Buus et al., 1998)

flexure would have been in this very point. It is related to the fact that relativist scales are not symmetrical, when sensations are increased and decreased as respects the reference value. Indeed, the loudness consecutively lowered twice, we should make use of the following equation:

$$
\frac{P_{0} \cdot 10^{\frac{L}{20}}}{P_{0} \cdot 10^{\frac{L_{i}}{20}}}=\frac{(1 / 2) \cdot \sqrt{1-1^{2} / C^{2}}}{1 \cdot \sqrt{1-(1 / 2)^{2} / C^{2}}}=\frac{\sqrt{C^{2}-1}}{\sqrt{4 \cdot C^{2}-1}} .
$$

It has turned out to be very difficult to draw a comparison between theoretical values and experimental data in the lower part of the curve. In one of the articles (Buus, Musch, and Florentine, 1998) the authors give a scheme of the curve that they empirically fitted for the area below $40 d B$ (See Figure 4)

Also the graph represents experimental points obtained by other authors. Many experimenters combine both the "ascent" method (a double increase in the subjective loudness) and the "descent" method (a double decrease in the subjective loudness) in their measurements. In doing this they consider the above methods equivalent as related to data

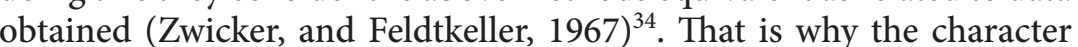
of location of experimental points in Figure 4 is simply inexplicable, issuing from the experiment logic. For example, it is unclear, how the researchers have received subjective loudness values above or below $1 / 2$, $1 / 4,1 / 8,1 / 16,1 / 32$ sones through the method discussed. It is also unclear, how it could turn out that Scharf and Stevens received subjective loudness points of $\approx 0.28$ and $\approx 0.47$ sone at practically equal correspondent values of sound pressure -31 and $32 d B$ (see Figure 4 ). The list of questions could go on.

Using our technique ("descent" method - Eq. (23)), we have made exact calculations of points for consecutive decrease in signal intensity, beginning from $40 d B$. The points received have been projected in Figure 4 (they are marked as A). It is clear that our points are quite well correlated with the general tendency of the experimental data received before. Yet they completely comply with the measurement technique, i.e. have values of $1 / 2,1 / 4,1 / 8,1 / 16,1 / 32$ sone.

Besides that, using our calculation method, we can try explaining the presence of groups of experimental points at $\approx 10, \approx 20, \approx 30, \approx 36$ $d B$, which do not "turn out" with the use of "descent" method (see Figure 4). For that we are using the "ascent" method (Eq. (22)) and the afore-calculated values of $A$ (received through the "descent" method), assuming that $\approx 16, \approx 27, \approx 34 d B$ correspondingly. Attention should be paid to the fact that $B$ points and reference $A$ points calculated through the "ascent" method have one and the same subjective loudness value in sones. And it is correct, because reference values of subjective loudness, i.e. subjective perception of certain levels of sound pressure, are the necessary condition for obtaining those points. It should also be noted that the calculated $B$ points have practically coincided with the groups of experimental points mentioned above. It serves an indirect proof to the fact that the researchers were resorting to both methods of "descent" and "ascent" in receiving experimental data, for they had considered these methods equivalent, which is not actually true.

\footnotetext{
${ }^{34}$ In reality it is not so (see Equations (23) and (24)). More than that, researchers often
} calculate the average value, using those techniques. 
It should be noted that Eq. (21) could be corrected concerning the low perception thresholds as follows:

$$
\frac{P_{0} \cdot 10^{\frac{L}{20}}-P_{0} \cdot 10^{\frac{L_{\min }}{20}}}{P_{0} \cdot 10^{\frac{L_{\mathrm{i}}}{20}}}=\frac{U_{\mathrm{H}} \cdot N / \sqrt{1-N^{2} / C^{2}}}{U_{\mathrm{H}} \cdot N_{\mathrm{i}} / \sqrt{1-N_{\mathrm{i}}^{2} / C^{2}}}=\frac{N \cdot \sqrt{C^{2}-N_{i}^{2}}}{N_{0} \cdot \sqrt{C^{2}-N^{2}}}
$$

where $L_{\min }$ is a threshold value of sound irritant intensity. Unfortunately, the low perception threshold can only be determined approximately, and its value lies in the confidence interval from 0 to $7 d B$ at the average value of $3 d B$ (Zwicker, and Feldtkeller, 1967).

It should be noted that there is little sense in the loudness curve as it is, since it simply connects points of different ranges, within which the comparison of sound loudness basically takes place. As an illustration, we have constructed four ranges, using Equation 4. Those ranges correspond to signal levels $L_{0}=40 \mathrm{~dB}, L_{2}=60 \mathrm{~dB}$ and $L_{4}=80 \mathrm{~dB}, L_{6}=100 \mathrm{~dB}$ (see Figure 5), that are used in traditional experimental studies.

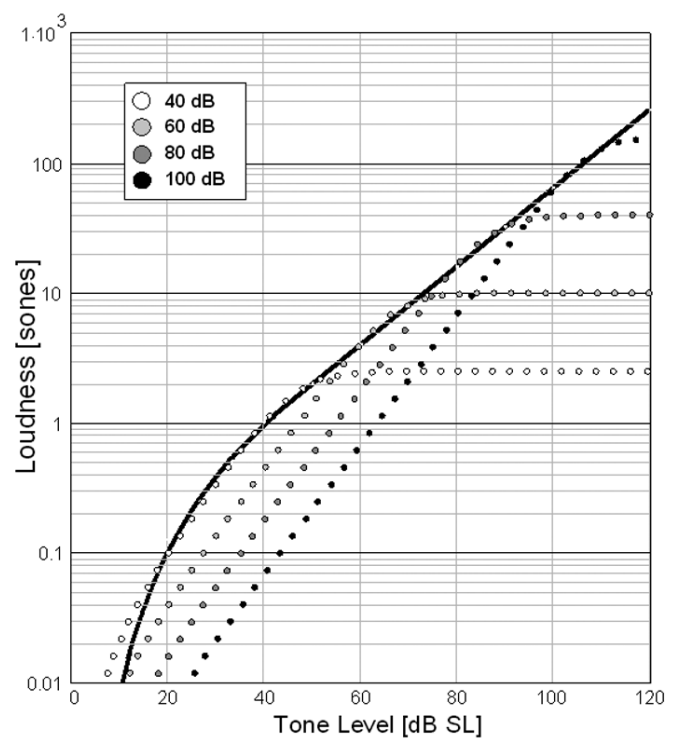

Figure 5. Loudness ranges. The continuous curve is the the ninth-degree polynominal regression, calculated with theoretical values of loudness obtained by Eq. (22) and Eq. (23). The doted lines signify loudness curves for the ranges that correspond to $L_{0}=40 \mathrm{~dB}, L_{2}=60 \mathrm{~dB}, L_{4}=80 \mathrm{~dB}, L_{6}=100 \mathrm{~dB}$
Calculated parameters for loudness ranges

Table 1

\begin{tabular}{ccc}
\hline $\begin{array}{c}\text { Signal intensity } \\
(L), d B\end{array}$ & $\begin{array}{c}\text { Subjective loudness } \\
(N), \text { sone }\end{array}$ & $\begin{array}{c}\text { Limit loudness value in the range } \\
(C), \text { sone }\end{array}$ \\
\hline 50 & 2 & 2,449 \\
60 & 4 & 4,344 \\
70 & 8 & 8,265 \\
80 & 16 & 16,203 \\
90 & 32 & $>10^{307}$ \\
\hline
\end{tabular}

Let us explicate the sense of the ranges received for the traditional technique of loudness doubling. Having the source level of sound field of $L d B$, the ear is tuned to the range from $N_{\min }$ to $N \cdot C$, where $N_{\min }=0$ sone (it corresponds to $L_{\min }$ - the lowest perception threshold within a given range), while $N \cdot C$ is the limit value of subjective loudness in sones for a given range. For example, the source level of sound field makes up $40 \mathrm{~dB}$, then the ear is tuned to the range from 0 sone to $(1 \cdot 2.449)$ sone, which corresponds to the values of sound irritant from $L_{\min } \approx 3 d B$ to $\approx 53 d B$ (see Figure 5) etc. Pay attention to the fact that $L_{\min }$, which corresponds to 0 sone, changes at transition into the next range (see Figure 5). And it is obvious, because the smaller the average loudness level, the weaker signals the ear can perceive, and vice versa. In case of adaptation, the sensory system can evidently tune to most informative frequency and dynamic ranges. One can check how the dynamic range changes not only 2 , but also 4,8 and 16 times, when it is necessary to distinguish signals. For these cases we have calculated $C$ for these ranges (see Table 1). It is clear from the table that $C \rightarrow \infty$, when it is necessary to compare the loudness of 32 sones with the reference level of 1 sone. It means that the maximum of dynamic range can not override $90 d B$, i.e., the sensory system is not able to trace such differences in the loudness level within one range .

It should be noted that, using our formulas, a researcher can construct the graphs of loudness relations for arbitrary values of subjective loudness and signal level. 
Thus, we have demonstrated that:

1. The formula that we have theoretically received, correctly describes the whole curve, unlike experimentally obtained formula that hold true only for separate segments of the curve;

2. Methods of "descent" and "ascent" are not equivalent in the measurements of subjective loudness, and that is why one should make use of relativist scales;

3. The loudness curve in psychophysics is not really continuous, but it simply connects segments of various ranges, within which the comparison of sound loudness values takes place.

\section{References}

Ackoff, R. (1999). Ackoff's Best: His Classic Writings on Management. New York: John Wiley \& Sons.

Bronshtein, I.N., and Semendyayev, K.A. (1980). Spravochnik po matematike dlya inzhenerov i uchaschihsya vtuzov [A Guide in Mathematics For Engineers and Technical College Students]. Moskva: Nauka.

Buus, S., Musch, H., and Florentine, M. (1998). On loudness at threshold. The Journal of the Acoustical Society of America, 104. P. 399-410.

Grimack, L.P. (1978). Modelirovanie sostoyanij cheloveka $v$ gipnoze [Modeling of Human States in Hypnosis]. Moskva: Nauka.

Heisenberg, W. (1989). Fizika i filosofiya. Chast' i celoe [Physics and Philosophy. Part and Whole]. Moskva: Nauka.

Landau, L.D., and Lifschitz, E.M. (1973). Teoreticheskaya fizika. Tom 2: Teoriya polya [The theoretical physics. Vol. 2: Field Theory]. Moskva: Nauka.

Louriah, A.R. (1973). Osnovy neyropsihologii [Foundations of Neuropsychology]. Moskva: Izdatel'stvo Moskovskogo universiteta.

Louriah, A.R. (1975). Oschuscheniya $i$ vospriyatie [Sensations and Perception]. Moskva: Izdatel'stvo Moskovskogo universiteta.

Pavlov, I.P. (1952). Polnoe sobranie sochineniy, 3, 2 [Complete Work, 3, 2]. MoskvaLeningrad: Akademiya Nauk SSSR.

Suprun, A.P. (2001). Psihosemanticheskiy metod issledovaniya mental'nosti [Psychosemantic Method of Studies In Mentality]. Obrazovanie v Sibiri (Spetsvypusk). P. 204-212.

Zwicker, E., and Feldtkeller, R. (1967). Das Ohr als Nachrichtenempfänger. Stuttgart: S. Hirzel Verlag. 